\title{
Digital holographic interferometry application on objects with heterogeneous reflecting properties
}

\author{
Jean-François Vandenrijt, Yuchen Zhao, Fabian Languy, Cédric Thizy, Marc P. Georges \\ Centre Spatial de Liège, STAR Research Unit, Liege Université, Liege Science Park, B-4031 Angleur, Belgium \\ Authore-mail address: mgeorges@uliege.be
}

\begin{abstract}
Some objects of industrial interest show zones which can be either scattering or specular. We present experimental digital holographic interferometry results obtained in a setup dealing with both at the same time. (c) 2018 The Author(s)

OCIS codes: (090.1995) Digital Holography; (090.2880) Holographic Interferometry ; (120.4290) Nondestructive Testing
\end{abstract}

\section{Introduction}

Digital holographic interferometry is a nondestructive testing technique which allows observing the full-field displacement of objects undergoing stress and/or movement. Usually the objects have surfaces with scattering properties, which allow forming easily an object beam at the recording sensor level. In the past, we already have shown the application of DHI in the case of objects with specular properties. In such case, specific illumination strategies need to be used, e.g. by using diffuser in order to generate artificial scattering in the object beam [1]. In some realistic cases, objects consist of separate parts with reflective properties that can be strongly different from their neighbors. For instance, specular parts can lie besides fully scattering ones. Spraying specular parts for a homogeneous scattering behavior is not always an option in real condition of NDT whereas simultaneous test results from both types of surface in the same field-of-view are often desired. In this contribution we show a DHI experiment with a metallic object with one part fully scattering and another one specular.

\section{Experimental setup and DH principle}

The particularity of our setup is the use of long-wave infrared $\mathrm{CO}_{2}$ laser $(10.6 \mu \mathrm{m})$ and microbolometer camera. It has already been presented for different nondestructive testing applications and owns several specific interesting properties [2,3]. At such long wave infrared (LWIR) wavelength, objects reflection is more specular than in visible, for a given roughness. Our motivation of using long wavelength is the large displacements that can be measured on space structures undergoing large thermal stresses $[1,4,5]$. Despite this, the setup proposed here can be applied at any wavelength.

Fig.1(a) shows the object developed for this demonstration experiment. First part (area A) consists of the polished metal which shows good specular reflectivity. In order to produce scattering surface on area $\mathrm{B}$, we have applied a white removable powder usually employed as developer for the so-called liquid penetrant method. Fig.1(b) shows the DH setup with two separate illuminations coming from the same laser. The first part is produced by the beam transmitted by beamsplitter BS2, folding mirror $\mathrm{M}_{\mathrm{A}}$ and illumination lens $\mathrm{L}_{\mathrm{A}}$. The beam illuminates a diffuser D. In a similar way to what was already presented elsewhere [1,5], the camera receives the light scattered by $\mathrm{D}$ and reflected by area A. The scattering area B is illuminated by a second part of the beam, reflected by BS2, mirror $\mathrm{M}_{\mathrm{B}}$ and expanded by lens $L_{B}$. Part of the light scattered by B reaches the camera sensor where it interferes with the reference beam.
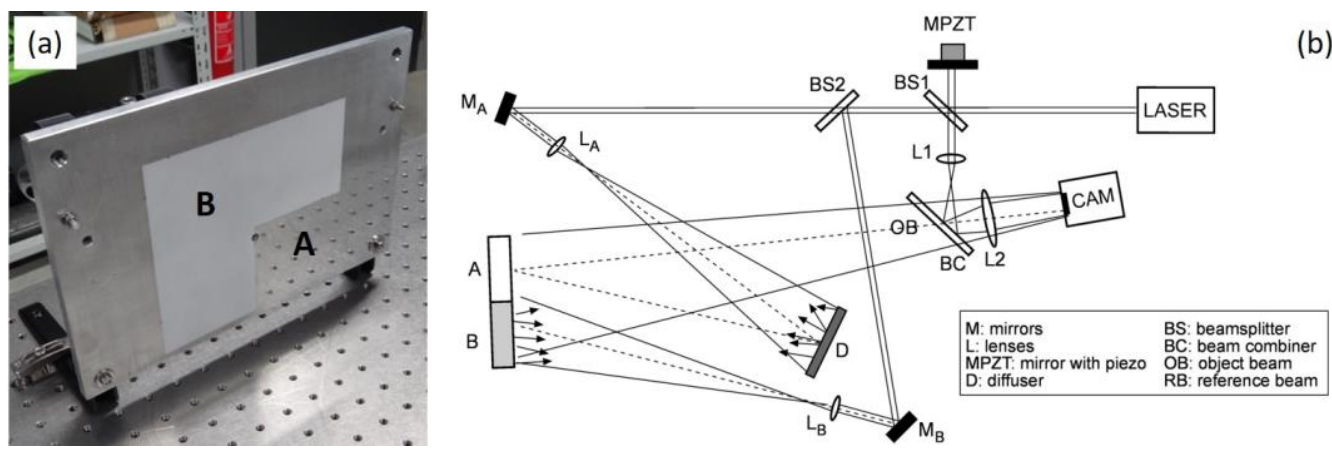

Fig. 1. (a) Object with two types of reflection properties: A specular, B scattering; (b) DH setup with two types of illumination 
It is possible to apply DH by performing the interference of the speckle object beam specularly reflected by area $\mathrm{A}$ and the light scattered by area B. In our case we use an in-line DH configuration with phase-shifting to benefit from the whole resolution.

\section{Results}

Fig. 2 shows the numerical reconstruction by phase-shifting DH in the in-line configuration. Fig. 2(a) shows the amplitude of reconstruction with both the specular area A, while scattering area B is surrounded in red. Fig. 2(b) shows the phase difference calculated numerically between two phases, before and after object deformation. The latter is induced by a mechanical device pushing at the back side of the object.

(a)

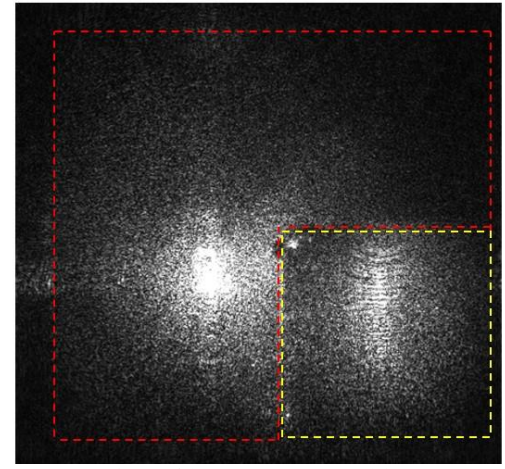

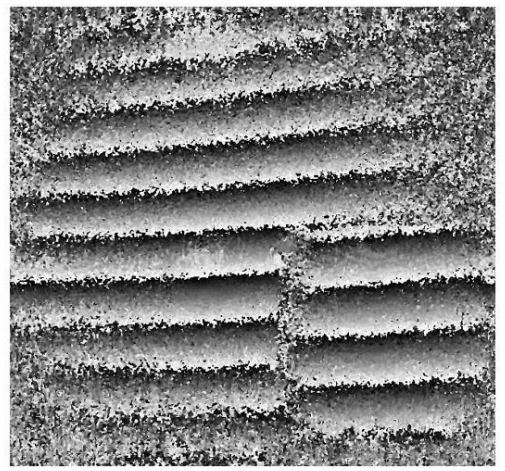

(b)

Fig. 2. Numerical reconstruction of heterogeneous object (a) amplitude and (b) phase difference after object deformation. Specular area A is surrounded in yellow and scattering area B in red.

Concerning the specular area $\mathrm{A}$, when the reconstruction distance is located at the specular plane, the fringes are localized on that surface [5]. This is also the case for the reconstruction of area B. One difference between the two object zones is the sensitivity vector, which relates the optical phase difference measured by DHI and the displacement. In the case of scattering objects, the sensitivity vector is the bisector of the illumination and the observation vectors. It depends on the setup geometry and the object extent. It varies between every object point observed. In the case of our setup, it is mainly oriented perpendicularly to the object surface, thus globally out-ofplane. In the case of purely specular objects illuminated by a diffuser, it was shown elsewhere that it is always outof-plane [6].

\section{Conclusion}

We have shown the DH is feasible on objects with two separate zones with either specular or scattering reflecting properties. Two distinct illuminations have to be used for dealing with each surface simultaneously. In DHI fringes of good quality are obtained and can be interpreted independently through their respective sensitivity vectors.

\section{References}

[1] J-F. Vandenrijt, C. Thizy, P. Queeckers, F. Dubois, D. Doyle, M.P. Georges, "Long-wave infrared digital holographic interferometry with diffuser or point source illuminations for measuring deformations of aspheric mirrors," Opt. Eng. 53 (11), 112309 (2014)

[2] M.P. Georges, J-F. Vandenrijt, C. Thizy, I. Alexeenko, G. Pedrini, B. Vollheim, I. Lopez, I. Jorge, J. Rochet, W. Osten, "Combined holography and thermography in a single sensor through image-plane holography at thermal infrared wavelengths," Opt. Exp. 22 (21), $25517-$ $25529(2014)$

[3] M.P. Georges, "Long-wave infrared digital holography," in New Techniques in Digital Holography, P. Picart ed. (John Wiley \& Sons, Inc., Hoboken, NJ, USA, 2015), Chap. 6

[4] J-F. Vandenrijt, C. Thizy, L. Martin, F. Beaumont, J. Garcia, C. Fabron, E. Prieto, T. Maciaszek, M.P. Georges, "Digital holographic interferometry in the long-wave infrared and temporal phase unwrapping for measuring large deformations and rigid body motions of segmented space detector in cryogenic test,” Opt. Eng. 55 (12), 121723 (2016)

[5] M. Georges, J.F. Vandenrijt, C. Thizy, Y. Stockman, P. Queeckers, F. Dubois, and D. Doyle, "Digital holographic interferometry with CO2 lasers and diffuse illumination applied to large space reflector metrology," Appl. Opt. 52 (1), A102-116 (2013).

[6] R.S. Hansen, "Deformation measurement of specularly reflecting objects using holographic interferometry with diffusive illumination," Opt. Lasers Eng. 28 (4), 259-275 (1997) 